\title{
Die Bedeutung einer reflexiven Didaktik für den bilingualen Unterricht
}

\section{Antonie Hornung}

Reflexivität - als wissenschaftlicher Habitus eingeführt durch den berühmten französischen Soziologen Pierre Bourdieu - ist für die Didaktik von unschätzbarem Wert: Die Lehrperson reflektiert ihr Handeln im Klassenzimmer, ihre eigene Person und nicht zuletzt die persönlichen Interessen im Feld Bildungsinstitution mit dem Ziel einer Optimierung des eigenen didaktischen Handelns. Diese Methode der Selbstevaluation von Unterricht erscheint für den bilingualen oder Immersionsunterricht von herausragender Bedeutung, da die Lehrperson, die hier im Sachfachunterricht für die Unterrichtskommunikation eine den Lernenden fremde Sprache benützt, zur Forscherin bezüglich der sprachlichen und der fachlichen Lernprozesse wird. Die Autorin zeigt in diesem Beitrag, ausgehend vom Kommunikationsmodell Karl Bühlers, zahlreiche mögliche Ansatzpunkte für eine reflexive Didaktik auf. Darüberhinaus unterstreicht sie den politschen Stellenwert der Sprachwahl für den Immersionsunterricht im Hinblick auf die Gefahr einer Unterschätzung der für die europäische Identität wichtigen großen Traditionssprachen.

\section{Sprachenpolitische und -didaktische Vorüber legungen}

Im gegenwärtigen sprachenpolitischen Diskurs auf dem (noch) plurikulturellen Spielfeld Europa scheinen sich zwei Positionen in antinomischer Ausrichtung zu profilieren: Zum einen wird Englisch als die lingua franca in Wirtschaft, Forschung und zunehmend auch in der Lehre gefordert und verteidigt, zum anderen wird Mehrsprachigkeit als ein Wert und als Reichtum gerühmt und ihre Förderung durch bildungspolitische Maßnahmen verlangt. Die Beherrschung - was auch immer man darunter verstehen mag - der Muttersprache plus Englisch gilt jedoch den VertreterInnen der letzteren Position nicht eigentlich als Realisierung des Ziels Mehrsprachigkeit, vielmehr wird von ihnen befürchtet, dass die zunehmende Dominanz des Englischen die alten Kultursprachen Europas zu Regionalsprachen und letztlich zu Dialekten herabwürdigt. (Vgl. hierzu den Über- 
blick über Neuerscheinungen zur europäischen Sprachenpolitik bei Altmeyer 2003).

Mit dem Gesamtsprachenkonzept von 1998 präsentiert die Schweizerische Konferenz der kantonalen Erziehungsdirektoren, den Gegebenheiten eines Landes mit vier Landessprachen und zahlreichen MigrantInnensprachen Rechnung tragend, einen Gegenentwurf zum English-only-Konzept und damit auch einen Entwurf, der als Modell für Europa taugen könnte: «Alle Schülerinnen und Schüler lernen, zusätzlich zur lokalen Landessprache, mindestens eine zweite Landessprache sowie Englisch; sie müssen darüber hinaus die Möglichkeit haben, eine zusätzliche Landessprache und allenfalls weitere Fremdsprachen zu erwerben.» (EDK, 1998, I Prinzipien und Zielsetzungen: 1).

Auf die Frage, ob und wie dieses Gesamtsprachenkonzept realisiert werden soll und welche Unterstützung die sogenannten Schweizer Minderheitensprachen Italienisch und Rätoromanisch genießen sollen, geben die Kantone allerdings höchst unterschiedliche Antworten. Während beispielsweise der Tabubrecher Zürich Englisch als erste fremde Sprache vor Französisch unterrichtet und darin viele Nachahmerkantone gefunden hat, stellt der Tessin mit der Anforderung der Viersprachigkeit (Italienisch-Französisch-Deutsch-Englisch) die Weichen eindeutig in Richtung "Schweizer Mehrsprachigkeit».

Künftig mindestens dreisprachige Bürgerinnen und Bürger auszubilden ist ein Ziel, das hohe Anforderungen an die Bildungsinstitutionen und die in ihnen Handelnden sowie die Ernsthaftigkeit aller Beteiligten auf die Probe stellt. Klar scheint zu sein, dass die Verwirklichung des Gesamtsprachenkonzepts durch Unterricht «alter Schule» kaum zu leisten ist.

Zum einen ist hier zu berücksichtigen, dass es die «alte Schule» der Vermittlung von Wissen und Können mit ihrer Zeit für ERKLÄREN - VERSTEHEN - (EIN)ÜBEN VON FERTIGKEITEN nicht mehr gibt. Nicht nur hat sich die Diversität der Fächer erhöht, was die Wochenstundenanzahl jedes einzelnen davon reduziert hat, sondern auch das jeweilige fachliche Wissen wächst in rasender Geschwindigkeit. Die Schulzeit wurde generell gekürzt (Matur nach 12 statt nach 12 1/2 bzw. 13 Schuljahren); die Zeit, die früher für die Vermittlung von Basiswissen und die Einübung in Schlüsselkompetenzen zur Verfügung stand, wird heute für den Erwerb von Spezialwissen und -fertigkeiten (man denke nur an die alle Fächer durchdringende Notwendigkeit der Informatikdidaktik!) benötigt. Anzunehmen, dass bei alledem nicht auch Gutes und Wichtiges verloren geht, scheint naiv.

Zum anderen ist zu bedenken, was aus der neurobiologischen Forschung an neuem Wissen über Lernen auf uns zukommt (Tomasello 1999/2002; Franceschini, Hufeisen, Jessner und Lüdi 2004). Lernen ist eine vernetzte Angelegenheit; für das Lernen und Erwerben von Sprachen gilt diese Erkenntnis erst recht. Stundentafeln und Lehrpläne, die den Unterricht in der Muttersprache und jedweder Fremdsprache strikt von einander trennen, repräsentieren heute noch ein behavioristisches Spracherwerbskonzept (Linke, Nussbaumer und Portmann, 
1994) Vernetzung ist gefragt, in der Organisation von Stundentafeln und erst recht für den Unterricht selbst. Wir brauchen Interdisziplinarität, aber nicht nur als gemeinsame und fachspezifisch getrennt abgehandelte Themenwahl, ${ }^{1}$ sondern verstärkt als fächerübergreifend koordiniertes didaktisches Handeln in der kommunikativen Situation Unterricht. Dass derartige Entwicklungen von der Basis aus und in Kenntnis der relevanten Forschungserkenntnisse vorangetrieben werden müssen, erklärt sich aus der zentralen Bedeutung der Erfahrung kommunikativen Handelns im Feld Schule für die Weiterentwicklung von Unterricht und in Hinblick auf dessen Erfolg. Weil Unterrichten über die Frage der fachlichen Inhalte und der Methoden ihrer Vermittlung hinaus als ein kontinuierlicher Kommunikationsprozess zu begreifen ist, werden im folgenden Beitrag auch Bezüge zum Kommunikationsmodell Karl Bühlers (Bühler 1934/99) hergestellt, das in besonderer Weise als geeignet erscheint, die neuralgischen Punkte der Kommunikation im Klassenzimmer auszuleuchten (Ehlich und Rehbein 1986), und wichtige Ansatzpunkte für eine reflexive Didaktik im bilingualen Unterricht aufzuspüren hilft.

\section{Die Vernetzheit kommunikativen Handelns im bilingualen Unterricht}

Der bilinguale oder Immersionsunterricht, in dem ein Sachfach in einer anderen als der Sprache der Region bzw. der Muttersprache der Lernenden unterrichtet wird, gilt weitherum als didaktischer Königsweg zu individueller Mehrsprachigkeit. (Brohy/Brégy 1998; Li Wei 2001) In der Schweiz und im europäischen Umland wird diese in Kanada geborene Form interdisziplinären Unterrichts inzwischen in zahlreichen Projekten aller Schulstufen und -typen praktiziert. ${ }^{2}$ In der Schweiz hat die Ermöglichung einer zweisprachigen Matur (Art. $18^{3}$ des MAR vom 16. Januar 1995) der Einführung bilingualer Unterrichtsmodelle auf Gymnasialstufe den Weg geebnet.

\section{Das Klassenzimmer als Ort kommunikativen Handelns ${ }^{4}$}

Jede Klasse oder Lerngruppe kann aufgrund ihrer zufälligen Zusammensetzung aus Individuen mit je einer persönlichen Geschichte als eine eigene social world (Strauss 1991) betrachtet werden, in der laut soziologischer Handlungsforschung immer wieder neue Aushandlungsprozesse anstehen: Vor einem gegebenen background gesellschaftlichen Verhaltens (Strauss 1978a; 1991) wird soziales Handeln in der Gruppe neu ausgehandelt, dies jedoch nicht beliebig, sondern nach bestimmten, durch die jeweiligen gesellschaftlichen Bedingungen vorgegebenen Mustern (Strauss 1978a; 1991, 213). Aushandlungsprozesse dieser Art können zu Veränderungen innerhalb der Gruppenstruktur führen. Den background stellen im sozialen Handlungsfeld Schule die institutionellen Gegebenheiten dar, also Lehrpläne, Lernziele oder auch Stundentafeln und Stunden- 
pläne, die Organisationsformen eben, die als für die Erreichung der Ziele erfolgreich betrachtet werden. Andererseits gehören zum background die innerhalb der Institution üblichen Handlungsweisen, beispielsweise unumkehrbares rollenspezifisches Verhalten von Lehrpersonen und Lernenden. Der aushandelbare foreground hingegen besteht in der in unterschiedlichen Schulsystemen und -fächern mehr oder weniger freien Lehrstoffauswahl (für exemplarischen Unterricht in der an Wissen überquellenden Wissensgesellschaft beispielsweise) und in der Entscheidung für bestimmte Wege der Vermittlung und des Lernens, also in Didaktik und Methodik. Dass dieser Bereich des foreground im Immersionsunterricht aufgrund der Vernetzung von Sachfach- und Sprachunterricht eine besondere Bedeutung gewinnt, erscheint naheliegend. 5

\section{Sachfachlehrpersonen sind auch Sprachlehrpersonen}

Lehrpersonen, die in Immersionsmodellen unterrichten, tun dies in der Regel aus Überzeugung und Passion; sie sind entweder selbst mehrsprachig aufgewachsen, oder sie haben längere Zeit im Land der neuen Unterrichtssprache gelebt, und sie glauben an den Spracherwerb durch Immersion. Ihr ureigenes Fach ist die Unterrichtssprache aber meistens nicht. ${ }^{6} \mathrm{D}$. h. sie begeben sich partiell auf didaktisches Neuland, dies in kommunikativer wie auch in fachlicher Hinsicht. Der Vorbereitungsaufwand ist groß, passendes Unterrichtsmaterial kaum vorhanden; vielfach müssen Lehrbücher im Land der Zielsprache gesucht oder unter großem Zeiteinsatz in diese übersetzt werden.

Unabhängig von den institutionellen und/oder politischen Interessen, die zur Entscheidung für ein Immersionsmodell beigetragen haben, sind selbst erfahrene Sachfachlehrpersonen beim Übergang zum bilingualen Unterricht mit einer Fülle neuer Aushandlungsprozesse konfrontiert. In einem solchen Feld die eigenen Motivationen und das sprachlich-didaktische Handeln kontinuierlich einer Reflexion zu unterziehen, erscheint als höchst sinnvoll, insbesondere aus linguistisch motivierten Gründen. Denn obwohl Immersionslehrpersonen in der Regel sprachfachlich Nichtfachleute sind - eine Tatsache, die gemeinhin auch als Qualität des Immersionsunterrichts betrachtet wird -, bewegen sie sich mit ihrem Sprachgebrauch doch auf Expertenterrain, und zwar im kulturell hochsensiblen Bereich der Pragmatik. Sie agieren in der Kommunikationssituation Klassenzimmer mündlich und schriftlich und haben sich dabei mit Fragen der Sprachrichtigkeit und Normativität auseinander zu setzen; sie benützen verschiedene Varietäten der fremden Sprache; sie führen einen interkulturellen Diskurs, was eine lediglich auf Sprachrichtigkeit zentrierte Interaktion im Klassenzimmer - man bedenke diesbezüglich die Problematik von Übersetzungen muttersprachlicher Sachfach-Lehrmaterialien in die Immersionssprache fragwürdig erscheinen läßt. Dies vor allem dann, wenn man den jugendlichen Lernenden mit der weiteren Sprache wirklich den Weg in die Kultur der anderen Sprache ebnen will. Kurz: Lehrpersonen stehen im bilingualen Unterricht in einem noch komplexeren Wirkungsfeld als im in der Muttersprache der Lernen- 
den erteilten. Sie haben die Ziele des written curriculum ihres Faches zu erreichen und tragen durch ihr sprachlich-kommunikatives Handeln via hidden curriculum zum Zweitsprach- und Zweitkulturerwerb bei.

\section{Reflexive Didaktik im bilingualen Unterricht}

\section{Reflexivität und Unterrichtspraxis}

Nicht jede Unterrichtsrealität kann durch aufwendige wissenschaftliche Evaluation überprüft werden, wohingegen eine regelmäßige kritische Hinterfragung des eigenen unterrichtlichen Handelns und seiner Ergebnisse in Form von Lernfortschritten der einzelnen Lernenden (Saudan 2003) durchaus praktikabel erscheint. ${ }^{7}$ Die Auswertung des eigenen Unterrichts durch die Lehrperson hat den Vorteil der unmittelbaren Umsetzbarkeit: Was die Lernprozesse negativ beeinflusst oder zu beeinflussen scheint, kann modifiziert oder weggelassen, was ihnen zuträglich ist, kann intensiviert werden. Dass solche Einschätzungen nicht frei sind vom Nachteil subjektiver Theorien im allgemeinen (Kallenbach 1996), liegt auf der Hand. Der Blick auf uns selbst vermag ohne blinden Fleck kaum auszukommen. Umso bedeutsamer erweist sich der Ansatz einer kritischen Reflexivität Pierre Bourdieus.

\section{Zum Begriff der Reflexivität}

Forschende im Feld verfolgen, so der französische Soziologe Pierre Bourdieu, bestimmte Interessen, die sich an ihre Position im Feld knüpfen, weshalb die eigene Person und ihre Interessen im Feld Gegenstand der Reflexion zu sein haben (Bourdieu/Wacquant 1996). Auch Lehrende bedürfen dieser Reflexivität, hegen doch auch sie aufgrund ihrer Position im Feld, d. h. innerhalb der Institution Schule, bestimmte Interessen, wie z. B. das Interesse, dauerhaft im Feld zu bleiben oder in ihm aufzusteigen, was ihnen den Blick auf ihr Handeln möglicherweise verstellt (Bourdieu 1982/90; vgl. hierzu und zum Folgenden auch Hornung 2002, Teil II).

Ins Zentrum der geforderten Reflexivität von Lehrpersonen rückt Bourdieu die von ihnen praktizierte Spracherziehung, da sie mit dieser zur symbolischen Herrschaft beitragen. Die Spracherziehung, die Bourdieu hier meint, zielt auf perfekte Sprachbeherrschung; sie ist durch zwei Eigenschaften charakterisiert: Die Eigenschaften, die die perfekte Sprachbeherrschung ausmachen, lassen sich mit zwei Wörtern zusammenfassen: Distinktion und Korrektheit (Bourdieu 1982/90: 38).

Damit ist ein Begriffspaar angesprochen, das vordergründig wenig mit Immersionsunterricht zu tun zu haben scheint, geht es doch in diesem gerade beim Sprachgebrauch in erster Linie um Verständigung und nicht um Korrektheit. Distinktion, also die sozial (de)klassierende Funktion eines bestimmten Sprachgebrauchs, ist aber gleichwohl im Spiel, und zwar zum einen, weil an verschiedenen Bildungsinstitutionen unterschiedliche Modelle (Alter; Anzahl Immer- 
sionslektionen; Immersionsfach/-fächer; Unterrichtsmethodik) praktiziert werden, und andererseits aufgrund der Wahl der Immersionssprache. Diese letztere ist, weil sprachliches Handeln im bilingualen Unterricht zum Erwerb der aus irgendwelchen Gründen präferierten fremden Sprache beiträgt, keineswegs irrelevant. Im Gegenteil, handelt es sich bei der Immersionssprache um eine zweite Schweizer Landessprache, so wird einer symbolischen Herrschaft zugearbeitet, der die Verständigung der Bürgerinnen und Bürger und das Verständnis für und unter einander ein Wert ist; anders, als wenn Mathematik oder Biologie auf Englisch unterrichtet wird, wo primär zu gelten scheint: Wer nicht im globalisierenden mainstream mitschwimmt, ist out.

\section{Reflexivität - Modelle für die (bilinguale) Unterrichtspraxis}

Unterrichtsrealität steht im Spannungsfeld zwischen Planung und Durchführung, aus der neue Planung entsteht. Die Einbindung der Reflexion über den erteilten Unterricht und über die eigene Rolle in ihm in den neuen Planungsprozess hilft, die Fokussierung auf Stoffvermittlung und Kompetenzenübung, also einen primär transaktional geplanten Unterricht, zugunsten des Einbezugs der Realisierbarkeit bestimmter Lernprozesse in einer gegebenen Gruppe von Lernenden in die Planungsarbeit zu verlagern. Eine solche Einschätzung sollte von innen erfolgen, denn, und dies wäre meine Hypothese zur Begründung der Notwendigkeit reflexiver Didaktik, und Hypothesen über das Verhalten einer Lerngruppe - die Lehrperson gehört zur Gruppe und ist Bestandteil der Reflexion werden für künftigen Unterricht dann eher planungs- und handlungsrelevant, wenn sie von einer Lehrperson formuliert werden, die sie kennt und in ihr handelt, als wenn sie von außen überstülpt werden.

In einem einfachen Modell läßt sich reflexive Didaktik wie folgt darstellen:

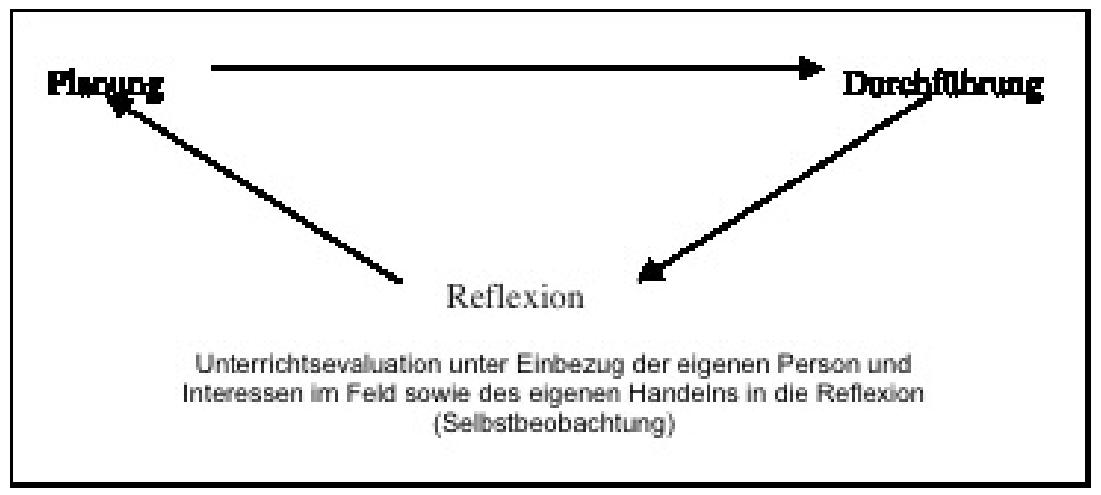

Figur 1: Einfaches Reflexitivitätsmodell (C) Antonie Hornung 2005 
Reflexion übernimmt in diesem Schema eine doppelte Funktion: Sie dient der Evaluation von Unterricht, und sie ist gleichzeitig Impuls für die unmittelbare Umsetzung von Erkenntnis. Die reflexive Betrachtungsweise stimuliert die Lehrperson zu konkreten Veränderungen des unterrichtlichen Handelns. Wird also die Frage: "Was geht gut in meinem Unterricht, und warum geht es gut?», aber auch: "Was geht nicht, und warum nicht?» ernst- und dauerhaft gestellt, trägt dies zur Schärfung der didaktischen Bewusstheit der Lehrperson und damit zur kontinuierlichen Unterrichtsentwicklung bei. Mit anderen Worten: Reflexivität wird alltägliche Praxis im Hinblick auf eine Optimierung des Unterrichts.

Schematisch lässt sich dieser Kreislauf als Handlungsquadrat zeigen:

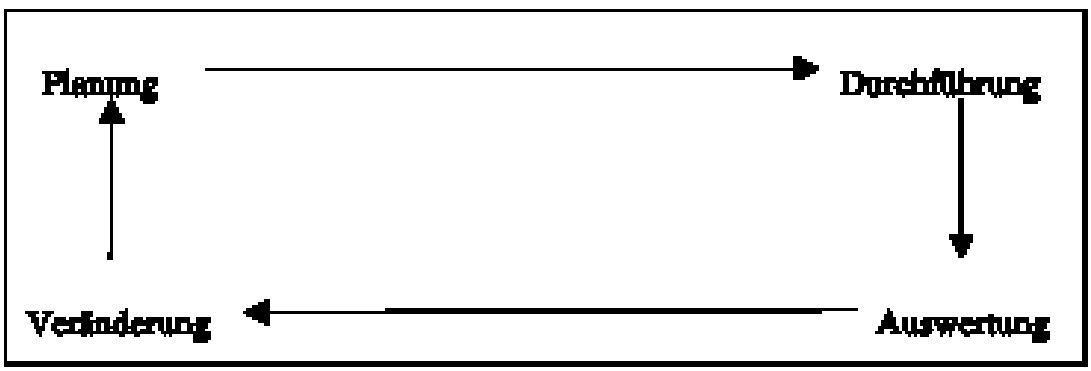

Figur 2: Modell reflexiven Handelns für den Unterricht (C) Antonie Hornung 2005

Gegenseitige Unterrichtsbesuche und der Austausch und die Diskussion subjektiver Theorien über den Erfolg oder Misserfolg von Unterricht ${ }^{8}$ gewährleisten mehr Objektivität und Verbindlichkeit. Deshalb bezieht das erweiterte Modell reflexiven Handelns für den Unterricht (vgl. Figur. 3) zusätzlich Kolleginnen und Kollegen in die Unterrichtsauswertung mit ein (vgl. hierzu auch Crookes 1993 und Altrichter 1998).

\section{Ansatzpunkte für eine reflexive Didaktik im bilingualen Unterricht}

Unterricht ist immer kommunikatives Handeln in asymmetrischer Kommunikationssituation. Demzufolge lässt er sich aus der Perspektive des Lehrens oder aus der Perspektive des Lernens analysieren. Dieser Unterschied kann nicht vernachlässigt werden, denn mit der unterschiedlichen Perspektive ändert sich auch die Zielrichtung der Beurteilung von Unterricht. Im ersteren Fall gerät, vor allem dann, wenn Unterrichten transaktional, also primär als Vermitteln von zu erlernendem Wissen bzw. als Trainingsraum für Könnensfähigkeiten verstanden wird, die Empfangsseite, im letzteren die Sendeseite der Kommunikation im Klassenzimmer ins Blickfeld der Kritik. D. h., wenn ich als Lehrperson auf meinen Unterricht blicke, denke ich womöglich zuerst an seinen Output, also an die - brillianten, guten, mittelmäßigen, ungenügenden - Leistungen meiner Schü- 
lerinnen und Schüler bezüglich Stoff und Fähigkeiten, während diese vermutlich, über Unterricht befragt, spontan viel eher über den Input, die Art der Vermittlung also und deren Echo bei ihnen selbst räsonieren werden.

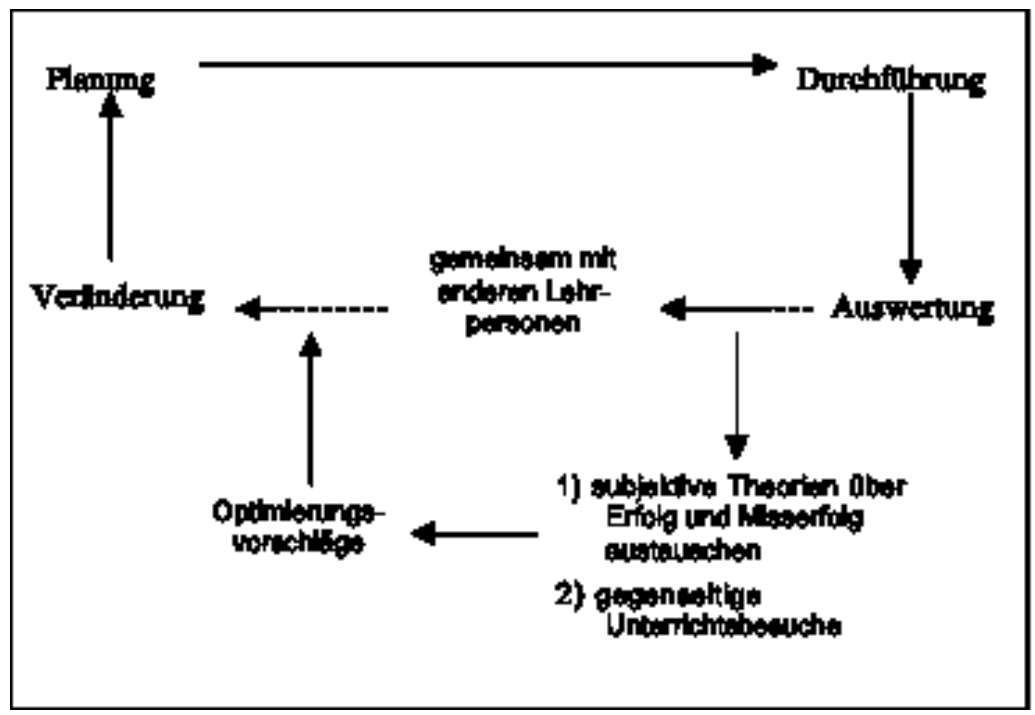

Figur. 3: Erweitertes Modell reflexiven Handelns für den Unterricht (C) Antonie Hornung 2005

Reflexive Didaktik nimmt beide Seiten und die Interaktion zwischen den beiden ins Visier. Sie überprüft Input und Output in ihrer wechselseitigen Beeinflussung, d. h. als zentrale Elemente kommunikativen Handelns im Klassenzimmer. Nach Karl Bühler sind die wesentlichen Parameter interaktiven sprachlichen Handelns Ausdruck, Appell und Darstellung (Auer und Bühler 1934/99). Versucht man seinen Blick auf die Funktionalität von Sprache im kommunikativen Feld auf den bilingualen Unterricht zu übertragen, so bietet sich der Reflexion darüber eine Fülle von Ansatzpunkten.

\section{Darstellung}

Darstellung bezeichnet nach Bühler «die Repräsentation eines Gegenstands oder Sachverhalts mittels eines Zeichens oder einer Zeichenkette» (Steiner 2004; Bühler 1934/99, bes. S. 28-33). Übertragen auf den bilingualen Unterricht, kann Darstellung für den gesamten Stoff plus die Sprache, mittels der er von der Lehrperson oder den Lernenden zum Unterrichtsgegenstand gemacht wird, stehen. Für die didaktische Reflexion lassen sich etwa die folgenden Ansatzpunkte herausarbeiten: 
- Stoffauswahl, Stoffaufteilung und Semesterplanung im Zusammenhang mit der zu verwendenden Sprache;

- Untergliederung des Stoffes in Unterrichtseinheiten;

- Organisation von Lernprozessen durch:

* Lehrvortrag,

* Einzel-, Paar- oder Gruppenarbeit verschiedenster Formen,

* schriftliche (Lesen und/oder Schreiben) oder mündliche (Hören und/oder Sprechen) Auseinandersetzung mit dem Lernstoff;

* weitere traditionelle und alternative Vermittlungsmethoden;

- Sprachgebrauch bei der Darstellung der Lehr-/Lerngegenstände:

* konsequente Einsprachigkeit vs. Übersetzen;

* Sprache im Textzusammenhang vs. Wortlisten;

* Texte vs. Charts (Schaubilder) und Bilder;

* Terminologie vs. alltägliche Wissenschaftssprache (Ehlich 1999);

* Korrektheit vs. Kommunikativität;

* Standardsprachgebrauch vs. Varietäten;

* Herkunftskulturorientierung vs. Zielkulturorientierung.

- Lernzielkontrolle als Prozesskontrolle (z. B. Portfolio oder Lernjournal);

- Gestaltung von Prüfungen.

\section{Ausdruck}

In Karl Bühlers Organonmodell steht die Bezeichnung Ausdruck für «die Beziehung zwischen Sender und dem Zeichen» (Steiner 2004; Bühler 1934/99, bes. S. 28-33). Damit tritt die jeweils einen Unterrichtsgegenstand darstellende Persönlichkeit, ihre Stoff- und Sprachbeherrschung, ihre Prosodie und ihr Akzent, ihr Umgang mit dem Unterrichtsstoff und der Unterrichtssprache ins Blickfeld der Reflexion. Alle im Klassenzimmer Anwesenden übernehmen diese Funktion, wenn sie sprechen oder sich schriftlich äußern, nicht nur die Lehrperson. Man denke diesbezüglich auch an die Rolle zwei- oder mehrsprachiger Klassenmitglieder, an Schülerinnen und Schüler, wenn sie Vorträge halten oder in Paar- und Gruppenarbeiten sich gegenseitig Lerninhalte erklären, wenn sie Fragen stellen oder auch Desinteresse demonstrieren. In diesen Bereich gehören auch die Nebenkommunikationen, d. h. halblaut geführte Gespräche von zumeist zwei Lernenden, die vom roten Faden der Unterrichtskommunikation abweichen, diesen aber auch ergänzen oder erklären oder im bilingualen Unterricht auch einer Worterklärung oder Übersetzung eines Sachverhalts dienen können, kurz: alle Formen verbaler oder nonverbaler Mitteilung als Ausdruck der jeweils sich mitteilenden Person.

Als Ansatzpunkte für die didaktische Reflexion des Kommunikationsverhaltens aller am Unterricht Beteiligten ${ }^{9}$ können hier dienen:

- Redehabitus (Aussprache und Prosodie):

* native-speaker-like vs. mit Akzent sprechen;

* Natürlichkeit des Sprechens vs. teacher's talk; 
* zusammenhängend vs. stichwortartig;

- Quantität der Redeanteile:

* dominant vs. zurückhaltend;

* lehrpersonzentriert vs. materialergänzt (Kassetten, DVD, Internet);

- Umgang mit der Normativität der Sprache:

* Spontaneität vs. Geplantheit;

* Sicherheit vs. Unsicherheit;

* Unsicherheitsakzeptanz (Lockerheit im Umgang mit Normverstößen) vs. Unsicherheitsvermeidung (Vermeiden nicht beherrschter Formulierungen);

- Umgang mit der Bilingualität:

* Mischsprache (Interlingua) vs. Sprachentrennung;

* Interferenzen vs. explizite Unterscheidung;

* Sprachkreationen vs. gelernte chunks;

- Umgang mit der Fachgebundenheit des Fremdsprachgebrauchs:

* Primat des Fachwissens vs. Übergewicht der Sprachnorm;

* Terminologie vs. Textualität;

* fachsprachliche Präzision vs. Umschreibung;

* Idiomatizität vs. näher an der Erstsprache der Lernenden orientierte und folglich leichter verständliche Formulierungen.

\section{Appell}

"Die Beziehung und auch die Wirkung, die das Zeichen auf den Empfänger ausübt» (Steiner 2004; Bühler 1934/99, bes. S. 28-33), bezeichnet Karl Bühler mit Appell. Im bilingualen Unterricht hat Sprache neben ihrer Darstellungsfunktion durch und durch Appellcharakter, denn sie soll als Ganzes gelernt werden, mehr noch eigentlich soll sie durch ihren Gebrauch und aus ihrem Gebrauch heraus erworben werden. $\mathrm{Ob}$ und wie gut dies gelingt, wird die Immersionslehrperson vermutlich ebenso interessieren wie der Lernerfolg im Sachfach. Inwieweit sie aber die Ergebnisse des Spracherwerbs, die Qualität der gelernten Sprache also, zum Gegenstand didaktischer Reflexion erheben will und kann, sollte ihrer Entscheidung anheimgestellt bleiben, hat für sie doch das Sachfach Priorität. Ausführliche Evaluationen des sprachlichen Fortschritts der Lernenden gehören auf jeden Fall in den Aufgabenbereich der Sprachlehrpersonen.

Über die verschiedenen lernziel- und leistungsbezogenen Evaluationsformen hinaus findet didaktische Reflexion bezüglich des Appellcharakters der Sprache im bilingualen Unterricht etwa die folgenden Ansatzpunkte:

- Verständnis der Unterrichtsinhalte:

* verbale, paraverbale und nonverbale Signale der Lernenden;

* Funktion von Nebenkommunikationen;

* Repetition vs. produktive Aneignung;

- kognitive und emotive Reaktionen:

* Aufmerksamkeit vs. Ablenkbarkeit bzw. Aufmerksamkeitsverweigerung; 
* fachliches Interesse vs. fachliches Desinteresse;

* Motivation vs. Demotivation;

* Solidarität vs. Wettbewerb;

- Teilhabe am kommunikativen Handeln:

* Aktivität vs. Passivität;

* Eigeninitiative vs. Aufgabenerfüllung;

* Mut zum sprachlichen Handeln vs. Sprechhemmung.

\section{Schlussfolgerungen}

Betrachtet man das Klassenzimmer als einen Ort kommunikativen Handelns, so ergeben sich, wie oben gezeigt, zahlreiche Ansatzpunkte für die didaktische Reflexion. Nachdenken über unterrichtliches Handeln fragt, ob die Prozesse im Klassenzimmer ablaufen wie erwünscht oder anders, ob das beobachtete Verhalten aller Beteiligten als für Lernen fruchtbar oder als schädigend empfunden wird, und es sucht Alternativen.

Immersionsunterricht fordert diese Art des Nachdenkens geradezu heraus, wird in ihm doch die Achtsamkeit zwangsläufig auf die fachlichen und auf die sprachlichen Lernprozesse gerichtet. Damit wird die Sensibilität für Lernprozesse ganz allgemein gestärkt; die Bereitschaft, den Unterricht umzugestalten, neue Methoden zu erproben und das didaktische Vokabular zu erweitern, wächst. Dieser Prozess der Umgestaltung bezieht die Lernenden, die in neuer Situation anders gefordert sind, voll mit ein.

Lehrpersonen, die sich auf bilingualen Unterricht einlassen, erleben in der Regel einen Rollenwandel: Von der Instanz, die Wissen vermittelt und sein Erlernen überprüft, werden sie zur Lernberaterin, zum Coach, die/der Lernprozesse initiiert, strukturiert und deren Verlauf beobachtet, aus dem Verlauf Schlüsse zieht und für das künftige didaktische Handeln fruchtbar macht. Hier setzt die reflexive Didaktik ein; die Lehrperson wird, indem sie ihren Unterricht selbst auf seine Erfolge und Misserfolge hin analysiert und zunehmend optimiert, zur Forscherin in einem Prozess, den sie selbst in Gang setzt und bei dessen Analyse sie die eigene Person nicht ausschließen kann. Dies umso mehr im bilingualen Unterricht, wenn der eigene Sprachgebrauchshabitus, das eigene kultur- und rollenspezifische Handeln via hidden curriculum mitbestimmend wird für die Lernprozesse im Klassenzimmer.

Inwieweit die Realitäten in den derzeit laufenden Immersionsprojekten einem solchen Idealbild von unterrichtlichem Handeln entsprechen, sollte von den in ihnen Tätigen beantwortet werden. Ihre Berichte jeweils an der März-Tagung der NordwestEDK oder an den APEPS-Tagungen im September jedenfalls deuten darauf hin, dass durch Anregung von außen und durch eine reflexive Didaktik der Lehrpersonen Veränderungsprozesse in Gang gekommen sind, denen weiterhin Erfolg zu wünschen ist. Wünschbar wäre Reflexivität aber auch in der Sprachenwahl. Sie täte uns gut. 


\section{Anmerkungen}

1 Diese Art von interdisziplinärem Unterricht findet beispielsweise statt, wenn man im Deutschunterricht Theodor Fontanes «Effi Briest» und parallel dazu im Französischunterricht Flauberts "Madame Bovary» liest, oder wenn in der Geschichte die französische Revolution behandelt und gleichzeitig im Deutschunterricht Georg Büchners «Dantons Tod» besprochen wird.

2 Informationen über Aktivitäten im deutschen Bereich sind zu finden über die hessische Mailingliste: bilingual@komm.bildung.hessen.de; ein Beispiel für ein Gymnasium mit doppelter Immersion (Englisch/Französisch) in Österreich ist die GIBS (Graz International Bilingual School); ein Immersionsmodell mit Deutsch als Immersionssprache führt das Liceo Galvani in Bologna, Italien.

3 Die von einem Kanton nach eigenen Vorschriften erteilte zweisprachige Matur kann ebenfalls anerkannt werden. (Reglement Nr. 400.710, 1995a: 7)

4 Eine ausführlichere Darlegung dieser Zusammenhänge findet sich bei Hornung (2002, $31 \mathrm{ff}$.$) .$

5 Das rasant wachsende neurobiologische Wissen über die Formen menschlichen Lernens stützt außerdem die Hypothese, dass Unterricht ohne Offenheit für derartige Aushandlungsprozesse letztlich weniger erfolgreich sein dürfte. Man vergleiche hierzu beispielsweise Tomasello (1999/2002, 15ff.); Franceschini, Zappatore und Nitsch (2003); Franceschini, Hufeisen, Jessner und Lüdi (2004).

6 Es sei denn, es wird, wie beispielsweise an der Graz International Bilingual School, die doppelte Facultas, also ein abgeschlossenes Studium im Sachfach und in der Immersionssprache, verlangt.

7 Verwiesen sei in diesem Zusammenhang einerseits auf die lebensgeschichtliche Relevanz der Notengebung für die Lernenden, andererseits auf die zunehmende Bedeutung von Portfolios für die Evaluierung von Lernleistungen in der allgemeindidaktischen Diskussion.

8 Die APEPS (Arbeitsgemeinschaft zur Förderung des mehrsprachigen Unterrichts in der Schweiz: www.plurilingua.ch) organisiert beispielsweise regelmäßig Tagungen, um Lehrkräfte landesweit zusammenzuführen und ihnen den Einblick in verschiedene Realitäten von Unterricht zu ermöglichen.

9 Der Fokus verschiebt sich, je nachdem die Lehrperson in ihrer Muttersprache unterrichtet oder die Unterrichtssprache auch für sie Fremdsprache ist. Dieselbe Unterscheidung gilt auch für die Analyse des Ausdrucks der Lernenden.

\section{Literatur}

Altmeyer, C. (2003). «Sprachkultur und Mehrsprachigkeit: Neuerscheinungen zur europäischen Sprachenpolitik (Teil 1).» Zeitschrift für interkulturellen Fremdsprachenunterricht $8 / 2 / 3,1-10$. [Online], [www.ualberta.cal-german/ejournal/Europaeische Sprachenpolitik.htm], [März 2004]

Altrichter, H \& Posch, P. (1998). Lehrer erforschen ihren Unterricht (3. Aufl.). Bad Heilbrunn: Julius Klinkhardt.

Auer, P. (1999). «Ausdruck - Appell - Darstellung (Karl Bühler). «In P. Auer, Sprachliche Interaktion. Eine Einführung anhand von 22 Klassikern (S. 18-29).Tübingen: Niemeyer.

Bühler, K. (1934). Sprachtheorie: Die Darstellungsfunktion der Sprache. Jena: Fischer. Neudruck 1999. Stuttgart: Lucius \& Lucius (UTB Wiss. 1159).

Brohy, Claudine / Bregy, Anne-Lore (1998): Mehrsprachige und plurikulturelle Schulmodelle in der Schweiz oder: What's in a name?. In: Bulletin suisse de linguistique appliquée. VALS/ASLA 67, S. 85-99. 
Crookes, G. (1993). «Action research for second language teachers: Going beyond teacher research.» Applied Linguistics, 13, 130-144.

Ehlich, K. \& Rehbein, J. (1986). Muster und Institution. Untersuchungen zur schulischen Kommunikation. Tübingen: Gunter Narr.

Ehlich, K. (1999). "Alltägliche Wissenschaftssprache.» In H. Barkowski \& A. Wolff (Hrsg.), Alternative Vermittlungsmethoden und Lernformen auf dem Prüfstand (S. 1-30). Regensburg: Fachverband Deutsch als Fremdsprache. (Materialien Deutsch als Fremdsprache 52).

Franceschini, R. , Zappatore, D. \& Nitsch, C. (2003). «Lexicon in the Brain: What Neurobiology Has to Say about Languages. In J. Cenoz, B. Hufeisen \& U..Jessner (Hrsg.), The Multilingual Lexicon. Dordrecht

Franceschini, R. , Hufeisen, B. , Jessner, U. \& Lüdi, G. (Hrsg.) (2004). Gehirn und Sprache: Psycho- und neurolinguistische Ansätze. Brain and Language: Psycholinguistic and neurobiological issues. Bulletin vals-asla, 78.

Hornung, A. (2002). Zur eigenen Sprache finden. Modell einer plurilingualen Schreibdidaktik. Tübingen: Niemeyer (RGL 234).

Kallenbach, C. (1996). Subjektive Theorien. Was Schüler und Schülerinnen über Fremdsprachenlernen denken. Tübingen: Narr.

Linke, A., Nussbaumer, M. \& Portmann, P. R. (1994). Studienbuch Linguistik (2. Aufl.). Tübingen: Niemeyer.

Li Wei (Hrsg.) (2001). The Bilingualism Reader. London and New York: Routledge.

Saudan, V. (2003). «Donner les moyens pour réfléchir, c'est donner les moyens pour agir. " Babylonia 3-4, 68-71.

Schweizerische Konferenz der kantonalen Erziehungsdirektoren (EDK) (1995a). Reglement über die Anerkennung von gymnasialen Maturitätsausweisen. (Maturitäts-Anerkennungsreglement, MAR) 400.710. Bern: [www.ag.ch/sarloutput/400-710.pdf], [März 2004].

Schweizerische Konferenz der kantonalen Erziehungsdirektoren (EDK) (1995b). Mehrsprachiges Land - mehrsprachige Schulen: Effizientes Sprachenlernen durch Sachunterricht in einer anderen Sprache. Bern: [www.edk.ch/PDF_Downloads/Dossiers/D33.pdf], [März 2004].

Schweizerische Konferenz der kantonalen Erziehungsdirektoren (EDK) (1998). Sprachenkonzept Schweiz. [www.romsem.unibas.ch/sprachenkonzept/Konzept.html], [März 2004].

Steiner, P., (2004). Karl Bühler: Das Organonmodell der Sprache. [http://santana.uni-muenster.de/Linguistik/user/steiner/semindex/buehler.html], [März 2004].

Strauss, A. (1978a). "General Considerations: An Introduction.» In A. Strauss, Creating Sociological Awareness (S. 209-221). New Brunswick/London: Transaction.

Strauss (1978b). «Paradigm and Prospects for a General Theory of Negotiation.» In A. Strauss, Creating Sociological Awareness Collective Images and Symbolic Representations. (S. 223230). New Brunswick/London: Transaction.

Strauss, A. (1990). «The Chicago Tradition's Ongoing Theory of Action/Interaction.» In A. Strauss, Creating Sociological Awareness Collective Images and Symbolic Representations. (S. 3-32). New Brunswick/London: Transaction.

Tomasello, M. (1999). The Cultural Origins of Human Cognition. Cambridge (Mass.) / London: Harvard University Press. / (2002). Die kulturelle Entwicklung des menschlichen Denkens. Frankfurt am Main: Suhrkamp.

Schlagworte: Immersion, zweisprachiger Unterricht, Mehrsprachigkeit, Reflexive Didaktik, Kommunikationsmodelle 


\section{Le rôle de la didactique réflexive pour l'enseignement bilingue}

\section{Résumé}

La réflexivité -méthodologie scientifique introduite par le célèbre sociologue Pierre Bourdieu - est d'une grande valeur pour la didactique: L'enseignant(e) peut améliorer son travail en devenant chercheur(e) en ce qui concerne son comportement communicatif dans la classe, sa personnalité et ses intérêts personnels dans le domaine de la formation. Cette méthodologie d'auto-évaluation semble encore plus efficace pour l'enseignement bilingue parce qu'il s'agit ici de viser deux objectifs: l'acquisition d'une langue étrangère et l'apprentissage du savoir et des habiletés disciplinaires.

Sur la base de la théorie sur la communication de Karl Bühler l'auteure explique les approches potentielles d'une didactique réflexive. En plus, elle souligne l'importance du choix de la langue pour l'enseignement bilingue, surtout par rapport au risque d'une dévalorisation des grandes langues traditionnelles européennes.

Mots clés: Immersion, enseignement bilingue, plurilinguisme, didactique réflexive, modèles de communication

\section{II valore della riflessività per l'insegnamento bilingue}

\section{Riassunto}

La riflessività - metodologia introdotta a livello scientifico dal celebre sociologo francese Pierre Bourdieu - ha una funzione importante per la didattica: l'insegnante riflette sul proprio agire in aula tenendo in considerazione la propria persona e i propri interessi e ciò con l'obiettivo di una ottimizzazione del proprio insegnamento. Questa metodologia di autovalutazione dell'insegnamento risulta estremamente valida per l'insegnamento bilingue, visto che l'insegnante, utilizzando una lingua veicolare per comunicare i contenuti della materia, si vede confrontata con i processi di apprendimento sia della lingua veicolare sia del sapere della materia. Sulla base della teoria dell'interazione linguistica di Karl Bühler, l'autrice dimostra una serie di possibili approcci ad una didattica riflessiva. Inoltre sottolinea l'aspetto politico della scelta linguistica per l'insegnamento bilingue, mettendo in rilievo il pericolo di una svalutazione delle grandi lingue tradizionali europee.

Parole chiave: immersione, insegnamento bilingue, plurilinguismo, didattica riflessiva, modelli di comunicazione 


\section{The Importance of Reflexivity in Bilingual Teaching}

Summary

Reflexivity has been introduced as a scientific habitus by the famous French Sociologist Pierre Bourdieu. This habitus is also extremely important for didactics: Teachers reflect on their acting in the classroom, on their own personality and last but not least on their concern in the field of education. This approach helps teachers to optimize their teaching. It is especially important for the practice of immersion-teaching, as it enables bilingual teachers to become researchers in the field of learning of subject-contents as well as in the field of language-acquisition. Basing her discussion on the theory of Karl Bühler, the author demonstrates some different approaches to this sort of applied research. Moreover, she indicates the political and cultural impacts of language-decision in immersionteaching, especially pointing out the dangerous under-estimation of traditional European languages when focussing on English-Immersion.

Key words: immersion, bilingual tuition, multilingualism, reflective didactics, communication models 
T h e m a 